\title{
Assessing Effects of Reduced Vision on Spatial Orientation Ability Using Virtual Reality
}

\author{
Max KINATEDER ${ }^{1,3}$, Emily A COOPER ${ }^{2,3}$ \\ ${ }^{1}$ National Research Council Canada, Canada \\ ${ }^{2}$ School of Optometry \& Helen Wills Neuroscience Institute, University of California, Berkeley, \\ USA \\ ${ }^{3}$ Note: Portions of this research were conducted when both authors were affiliated with the \\ Department of Psychological \& Brain Sciences, Dartmouth College, USA
}

ORCID 0000-0002-1669-3900, ORCID 0000-0003-4889-7446

max.kinatederanrc-cnrc.gc.ca, emilycooperaberkeley.edu

\begin{abstract}
How vision loss affects spatial orientation is relatively unknown. We investigated how simulated reductions in central vision and visual field influence spatial orientation in Virtual Reality. Participants were assigned to three groups: control (typical vision), reduced central vision (low acuity and contrast sensitivity) and reduced field (restricted peripheral vision). Participants were disoriented within a virtual room, and reoriented themselves towards a remembered target. In Experiment 1, we manipulated the shape and features of the room. In Experiment 2, we manipulated the lighting of the room. We measured response times and re-orientation precision. In Experiment 1, the reduced field group was slower and less precise to reorient; all groups were slower when presented with conflicting cues. In Experiment 2, the reduced field group was again slower than the other groups, but we did not observe a difference in precision. When environment lighting was dynamic, all groups were slower to reorient.
\end{abstract}

Keywords: Spatial Orientation, Low Vision, Virtual Reality.

\section{Introduction}

Independent navigation is an important activity in daily life, and one that is negatively impacted by visual impairment. The global prevalence of visual impairment is in the tens of millions of people, so developing new strategies and tools that can successfully support navigation for these individuals is a high priority (Bourne et al., 2017; Chan et al., 2018). The majority of people with impaired vision are not functionally blind, and thus research has focused on how to enhance or supplement visual information available in the environment (Weiner et al., 2018). Many causes of visual impairment either primarily affect central vision (causing reduced visual sensitivity near the point of fixation) or primarily affect peripheral vision (causing a restriction of the visual field). Understanding the effects of central and peripheral vision loss on complex visually- 
guided behaviors is important for developing navigation aids; however, many aspects of the basic perceptual and cognitive processes that underlie successful navigation are still poorly understood (Burgess, 2006; Epstein et al., 2017).

Prior work investigating navigation with impaired vision has examined a range of tasks and employed a combination of participants with simulated and genuine visual impairments. Overall mobility performance is impaired by even moderate peripheral field loss, as well as reductions in central acuity and contrast sensitivity (Black et al., 1997; Brown et al., 1986; Hassan et al., 2002, 1999; Kuyk and Elliott, 1999; LovieKitchin et al., 2010; Marron and Bailey, 1982; Turano et al., 2004). Acuity refers to the ability to resolve visual detail, whereas contrast sensitivity refers to the ability to detect changes in light intensity. Spatial learning, which is an important cognitive aspect of navigation, may only be impaired with severe vision reduction, if at all (Barhorst-Cates et al., 2017, 2016; Legge et al., 2016a, 2016b). However, the increased attentional demand required to navigate with impaired vision can impact learning (Rand et al., 2015). Spatial orientation refers to knowing one's location and heading direction in the environment (e.g., where are important objects and structures around me? How do I need to move to get to my goal?). While the phenomenology and mechanisms of spatial orientation are actively studied in cognitive science and neuroscience, relatively few studies have examined the impact of visual impairment on spatial orientation in a controlled setting (Daga et al., 2017; Kalia et al., 2008). One study found that vision loss did not affect spatial orientation (Kalia et al., 2008). Another study suggested that reduced peripheral vision increased the time required for orientation, but the task also required simulating mobility in Virtual Reality (Daga et al., 2017). The mobility aspect of the task could have impacted response time even if spatial orientation was unaffected. Thus, at present more investigation is needed to understand whether and how visual impairment impacts the processes that support spatial orientation during real-world navigation.

To address this gap, we adapted a sensitive behavioral paradigm with minimal mobility or spatial learning requirements in order to characterize the impact of reduced vision on spatial orientation (Cheng et al., 2013). We use the 'room re-orientation' paradigm, which was first introduced to study animal behavior in the 1980's and has since been extensively used in human development and cognition (Cheng, 1986; Cheng et al., 2013; Hermer and Spelke, 1994; Ratliff and Newcombe, 2008). The basic paradigm is illustrated in Figure 1. A person is tasked to use their vision to reorient themselves after they are disoriented and placed back in a familiar environment. Multiple possible cues/strategies are possible during reorientation. For example, adults with typical vision use geometric environment cues (e.g., relative locations of walls, corners, or openings) and non-geometric cues (color, texture, and lighting), but children tend to disregard the non-geometric cues (Hermer and Spelke, 1996).

In two experiments, we use a Virtual Reality (VR) version of the room re-orientation paradigm to quantify the effect of simulated reductions in vision on the precision of reorientation, the time it takes to reorient, and the re-orientation strategy used by adults. We examine these effects in a range of virtual room types and under two lighting conditions. Building on prior modeling methodologies, we also formulate a probabilistic model that can accommodate arbitrary target locations, environmental geometries, and cue selection (Cheung et al., 2008; Stürzl et al., 2008; Xu et al., 2017). Our results suggest that reductions in visual field can both increase the time needed to reorient and reduce the precision with which people reorient, while reductions in central vision - at 
least to the degree simulated here - did not significantly affect these variables relative to the control group with typical vision.

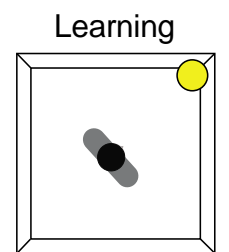

Target is seen by participant in a room

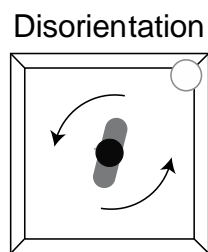

Participant is disoriented, target hidden



target was

Fig. 1. Illustration of the room re-orientation paradigm. A top-down view is shown of a person standing in the middle of a room. During a learning phase (left panel), they learn the spatial location of a target (yellow circle). Next (middle panel), they are disoriented, for example, by spinning with their eyes closed. Lastly (right panel), they open their eyes and are asked to respond where the target, which is now hidden, was originally located. Various cues may be available for this task, such as the shape of the room and the presence of objects or features on the walls (see Methods section for details for each experiment).

\section{Experiment 1}

\subsection{Methods}

Participants. Thirty adults participated, all with normal or corrected-to-normal visual acuity (0.00 logMAR or better). Participants were pseudo-randomly assigned into three groups ( $\mathrm{n}=10$ per group). The control group (mean age: 19.30 years, 7 female) completed the experiment without any simulated visual impairment. The reduced central vision group (mean age: 22.90 years, 6 female) wore a pair of swim-goggles modified binocularly with Bangerter occlusion foils (two layers of LT 0.1 foils; Ryser Optik, St. Gallen, Switzerland). These goggles reduce optotype acuity to approximately 1.3 $\log$ MAR and reduce contrast sensitivity by approximately one log unit (Mars test) (Huang et al., 2019). We refer to this group as reduced central for brevity. The reduced field group (mean age: 19.00 years, 8 female) wore swim-goggles modified with opaque electrical tape that covered the left eye completely and left a field of view of approximately $20^{\circ}$ in the right eye. All participants gave written informed consent and were compensated for their time. The procedures were approved by the Dartmouth College Institutional Review Board and complied with the Declaration of Helsinki. Four participants were excluded because debriefing interviews revealed that they did not follow the instructions (four additional participants were recruited to complete the sample size of $n=10$ per group).

\subsection{Apparatus}

Participants performed the experiment while standing in an unobstructed area and wearing a head-mounted display that presented them with a virtual $3 \mathrm{D}$ environment (Rift CV1, Oculus, Irvine CA). The display system had a wide FOV $\left(\sim 100^{\circ}\right.$ diagonal $)$ and a resolution of 1080 x 1200 pixels per eye. Stimuli were generated using the Unity 2017.3 
software package (Unity Technologies, San Francisco CA). Head location and rotation (roll, pitch, and yaw), derived from three infrared trackers and the built-in accelerometer, were used to update the display and track the orientation of the participant's head as they looked around the virtual environment. Participants used a handheld controller to indicate responses.

\section{A}

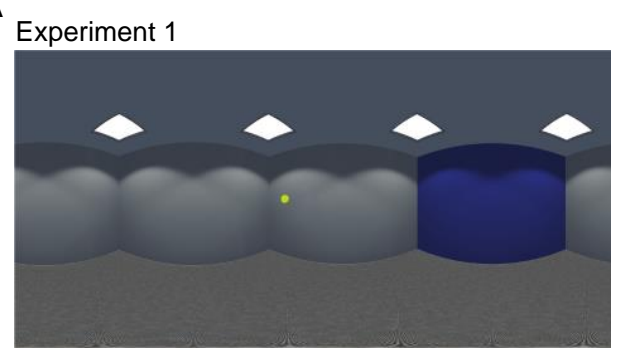

B

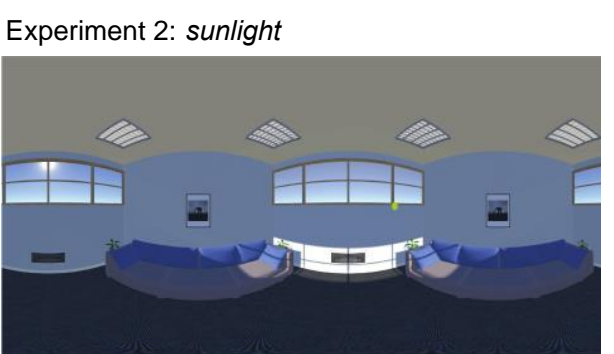

Experiment 2: ceiling lights

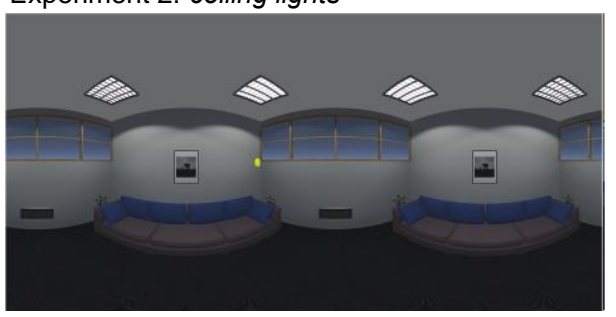

Fig. 2. Panoramic images of the virtual rooms. A) In Experiment 1, the rooms had solid walls, a textured floor, and standard overhead lighting. In some conditions, one of the walls was colored blue. The reorientation target (a gold coin) was visible at eye level. B) In Experiment 2, the rooms had additional furniture and windows, placed symmetrically along two walls. The virtual lighting was either from sunlight through the window or via the ceiling lights. Image values are adjusted for visibility in the figure.

\subsection{Task}

Each trial consisted of the 3 stages (see Figure 1 for an illustration of the task):

(1) Learning: Participants were teleported into the center of a room and tried to memorize the location of a gold coin $\left(4.8^{\circ}\right.$ in diameter, located at eye level; Figure $2 \mathrm{~A}$ shows an example panoramic image of one virtual room and coin). The coin was positioned at a yaw selected at random from a uniform distribution and the participants always teleported such that they were initially facing the coin. The coin was visible for 10 s during which participants were encouraged to look around the room for context and 
remember the coin's location. They were also instructed that the room may have changed in appearance when they returned (see Experimental conditions below).

(2) Disorientation: Participants were immersed into a random starfield with no salient visual features. They were instructed to turn in place and search visually for a green circular target, located approximately at eye level $\left(4.8^{\circ}\right.$ in diameter $)$ at a random yaw. Once the participant found the target, it disappeared and reappeared at another random yaw. This procedure was repeated three times. After the fourth time, participants moved to the next stage.

(3) Test: Participants were teleported back into the room at a new, random yaw. Their task was to turn towards the location where the coin was placed during the learning phase and to press the controller button. The heading direction was then recorded, as well at the response time.

Prior to beginning each trial, the disorientation procedure was repeated to make sure participants did not orient relative to the physical laboratory space or the virtual room from the previous trial. After completing all trials participants filled out the Santa Barbara Sense of Direction Scale (SBSOD) (Hegarty et al., 2002).

\subsection{Conditions}

Each participant completed 12 trials in each of five different conditions (60 trials total) presented in pseudo-randomized order (Figure 3). As in prior work (Hermer and Spelke, 1996, 1994), one condition included a rectangular room (4m wide, $8 \mathrm{~m}$ long, $3 \mathrm{~m}$ high) with no features, decorations, or windows (rectangular room), and a second condition
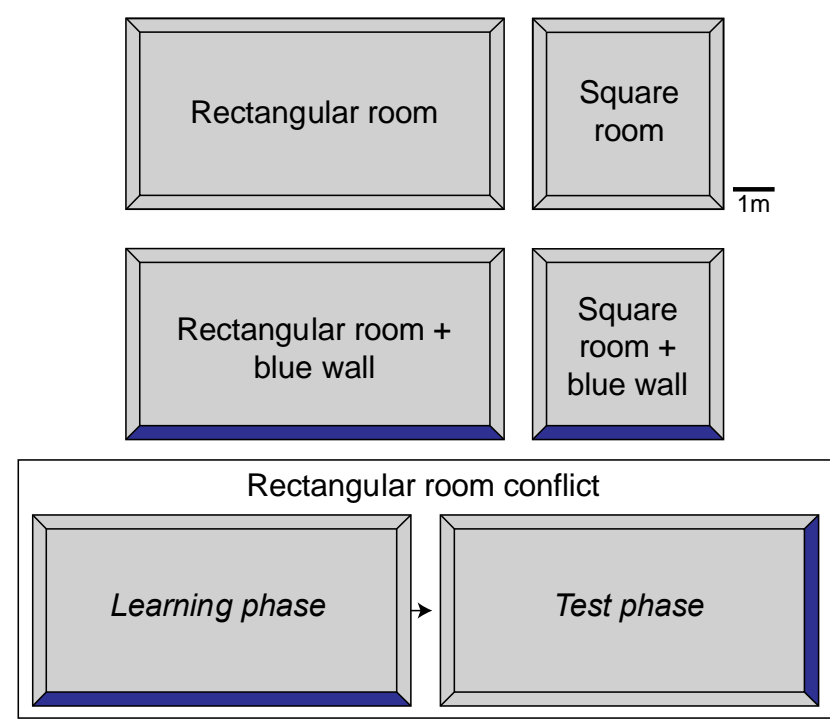

Fig. 3. Illustrations of the virtual rooms and conditions in Experiment 1. Each room is illustrated with a top-down view. In the blue wall conditions, the blue paint could appear on any wall. In the rectangular room conflict condition, the painted wall moved randomly either leftward or rightward between Learning and Test phases.

had the same room shape, but a large salient feature was added (one wall with blue paint), which could serve as a landmark to disambiguate geometrically identical 
locations (rectangular room + blue wall). In a rectangular room conflict condition, the location of the blue wall rotated by $90^{\circ}$ between the Learning and Test phases, forcing participants to decide whether or not to use this cue as a landmark. The remaining two conditions - square room and square room + blue wall-were identical to the first two, but the room width and length were both $4 \mathrm{~m}$. All rooms were illuminated from virtual ceiling lights.

\subsection{Data analysis}

Analyses were performed using Matlab (v2018b) and R (v3.5.1). First, we confirmed with a one-way ANOVA that there were no significant differences between the three experimental groups in the $\operatorname{SBSOD}\left(\mathrm{F}(2,27)=2.14, \mathrm{p}=.137, \eta_{\mathrm{p}}^{2}=.14\right)$. The average score was $4.45(\mathrm{sd}=0.75)$. The analyses for this experiment were conducted in an exploratory manner and used to develop a new probabilistic model to describe reorientation behavior; the final model and analysis pipeline were then used to analyze Experiment 2.

The amount of time taken to re-orient on each trial was calculated as the duration between the start of the Test phase and the time when the participant pressed the button. More than half of all responses were made within 5.5 seconds. Trials in which the response time exceeded 45 seconds ( 99.7 percentile, 7 trials out of 2400 trials) were assumed to be mistrials (i.e., participant took a break or became confused about which phase they were in) and were excluded. The response time for each participant in each condition was calculated as the median across the included trials.
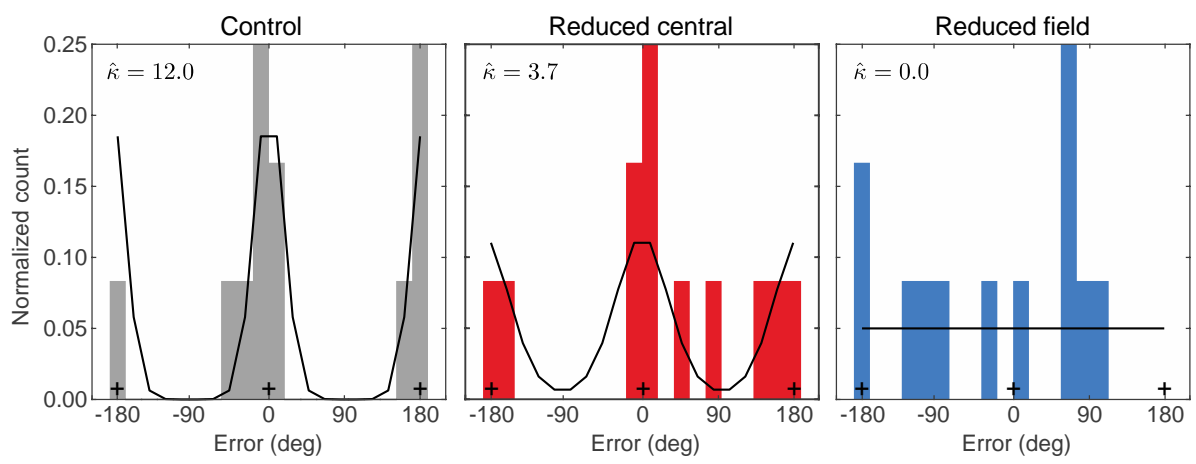

Fig. 4. Example angular errors and model fits. Each panel includes a histogram of the angular response errors (in degrees) made by one participant in each group during the rectangular room condition. In this condition, $0^{\circ}$ and $+/-180^{\circ}$ are geometrically accurate responses. Black lines indicate the probability associated with each error bin for the best-fit von Mises response model (concentration parameters are indicated for each participant).

To assess the pattern of re-orientation performance, we next computed the angular response error on every trial by calculating the circular distance between the target's true orientation and a participant's heading orientation when they pressed the button. Errors are represented on a scale of $+/-180^{\circ}$, with negative values indicating clockwise and positive values indicating counterclockwise errors. Circular calculations were performed using the Matlab circstats toolbox (Berens, 2009). Example histograms of the angular 
response errors of three participants (one from each group) during the rectangular room condition are show in Figure 4. Geometrically identical errors are indicated with a ' + '. In the examples shown, these errors include $0^{\circ}$ and $+/-180^{\circ}$ because each point in a featureless rectangular room contains a geometrically identical point at a $180^{\circ}$ rotation. In the example histograms, the control and reduced central participants made the majority of their errors near geometrically identical points, whereas the reduced field participant made more uniformly distributed errors.

Next, we wanted to quantify how effectively participants were using environmental information to reorient themselves. We defined a hypothesized distribution of errors via a continuous circular probability density function. For the rectangular room and square room conditions, we assumed that the probability of errors was highest at geometrically identical locations (that is, responses $0^{\circ}$ and $180^{\circ}$ relative to the target in the rectangular room and responses $0^{\circ}, 90^{\circ},-90^{\circ}$, and $180^{\circ}$ relative to the target in the square room). However, the spread of the errors around each location for each participant was unknown and was left as a free parameter. Specifically, the probability of a given angular response error $(\theta)$ was specified as a normalized sum of multiple von Mises distributions. The von Mises distribution is analogous to the Gaussian distribution on a circle (with all angular quantities specified in radians). The model can thus be written as:

$$
p(\theta)=\frac{1}{n_{a}} \sum_{a \in A} \frac{e^{\kappa \cos (\theta-a)}}{2 \pi I_{0}(\kappa)}
$$

where $\kappa$ denotes the von Mises concentration parameter, $I_{0}$ denotes a modified Bessel function of the zeroth order, $a$ denotes the angle in radians to geometrically equivalent locations contained in set $A$, and $n_{a}$ denotes the number of elements in $A$. Converting angles from radians to degrees, $A=\left\{0^{\circ}, 180^{\circ}\right\}$ in the rectangular room condition and $A=$ $\left\{0^{\circ}, 90^{\circ},-90^{\circ}, 180^{\circ}\right\}$ in the square room condition. For each von Mises, $a$ is analogous to the mean of the Gaussian and the concentration parameter $\kappa$ is inversely related to the variance (higher $\kappa$ values correspond to tighter distributions, and vice versa). Thus, $\kappa$ served as our measure of the precision of responses.

We used the maximum likelihood method to fit the responses of each participant with this model, where the likelihood was represented by $\mathrm{p}(\{\theta\} \mid \kappa)$, and $\{\theta\}$ denotes the set of angular errors measured for a given participant. For each participant we performed a parameter sweep of $\kappa$ from 0 to 300 in steps of $1 / 3$. We denote the concentration of the maximum likelihood model as $\hat{\kappa}$, and refer to this as the response precision. For each example participant in Figure 4, a black line illustrates the best fit model overlaid on the error histograms, and response precision values are indicated. For the reduced field participant, the best fit model is a uniform response, which occurs when $\hat{\kappa}=0$. These precision values provide a quantitative way to compare the reorientation performance of the different groups, while allowing us to adopt a continuous response variable.

This model was slightly elaborated for the remaining three conditions, which contained a salient color feature in addition to room geometry information (rectangular room + blue wall, square room + blue wall, and rectangular room conflict). Specifically, the von Mises model was extended to incorporate an additional cue: 


$$
p(\theta)=\left(1-w_{b}\right)\left(\frac{1}{n_{a}} \sum_{a \in A} \frac{e^{\kappa \cos (\theta-a)}}{2 \pi I_{0}(\kappa)}\right)+w_{b}\left(\frac{1}{n_{b}} \sum_{b \in B} \frac{e^{\kappa \cos (\theta-b)}}{2 \pi I_{0}(\kappa)}\right)
$$

Here, $b$ denotes the angle to the blue wall. In the current experiments, $B=\left\{0^{\circ}\right\}$ and $n_{b}=1$ for all three conditions, because there was only one blue wall in the room. In addition, $0 \geq w_{b} \geq 1$, which denotes the weight given to the wall in determining the reorientation response. For example, if the introduction of the salient feature eliminated geometric response errors, $w_{b}=1$. If individuals still made some geometric mistakes on some trials, $w_{b}<1$. Another possible scenario is that people perform a weighted cue combination on each trial. Because the cue conflict was large, prior work suggests that people will pick a single cue in the current paradigm (Twyman et al., 2018). Note that when $w_{b}=0$, Equations 1 and 2 are identical.

To analyze the differences between groups and conditions, we compared the response precision and response times using ANOVAs with condition as a within subjects variable, and group as a between subjects variable. Initial examination revealed that the fit residuals were not normally distributed, so we used permutation tests to calculate $p$ values for each of the main effects and interactions using the $1 \mathrm{mPerm}$ package in $\mathrm{R}$ (maximum iterations $=10000$, stopping if standard error of the estimated $\mathrm{p}$ values was lower than $0.01 * \mathrm{p}$ ) (Wheeler and Torchuano, 2016). In most cases, the use of permuted $\mathrm{p}$ values did not affect any conclusions regarding statistical significance, or produced more conservative interpretations. We denote permuted $\mathrm{p}$ values as $\mathrm{p}_{\mathrm{p}}$. Followup tests were performed using Dunn's test for pairwise comparisons (Dinno, 2017), which is a non-parametric test for determining statistically significant differences in medians. Data and example code for both Experiments are available in a publicly accessible repository (https://github.com/eacooper/SpatialOrientationVR_SpatialCog).

\subsection{Results}

Reorienting using room shape. To determine how well participants could use the layout of a room alone to reorient themselves, we first examine the conditions without a salient feature (i.e., square room and rectangular room). Figure 5A shows the response precision $(\hat{\kappa})$ for each participant in each group and condition. Participants with reduced field tended to have lower precision, which in some cases were very near zero, or equivalent to guessing, whereas participants in the other two groups had higher precision. A 3x2 ANOVA showed a main effect of group $\left(\mathrm{F}(2,27)=5.52, p_{p}=.041\right.$, $\eta_{\mathrm{p}}^{2}=.29$ ), and follow up tests showed that the reduced field group (median $=2.84$ ) had significantly lower precision than the control group $($ median $=11.50, z=3.75, p<.001)$ and the reduced central group (median $=5.67, z=2.24, p=.025$ ). While the response times in the reduced field group were descriptively longer than the other two groups (Figure 5B), an ANOVA showed that the main effect of group did not reach statistical significance $\left(\mathrm{F}(2,27)=2.56, p_{p}=.096, \eta_{\mathrm{p}}^{2}=.16\right)$. Follow-up tests, however, showed significantly slower response times in the reduced field group (median $=6.91 \mathrm{~s}$ ) than in the control group (median $=4.30 \mathrm{~s}, z=3.42, p<.001$ ) and in the reduced central group (median $=4.74 \mathrm{~s}, z=2.71, p=.006)$. These results suggest that reduction in visual field can increase the challenge of reorienting oneself using layout information. However, the rooms in these two conditions were very simple, so next we examined reorientation performance with the addition of a large, salient visual feature. 
Reorienting with a salient feature. Across all conditions, we observed descriptively lower precision in the reduced field group (Figure 6A), however this was not statistically significant in the ANOVA $\left(\mathrm{F}(2,27)=4.01, p_{p}=.061, \eta_{\mathrm{p}}^{2}=.23\right)$. Follow up tests between groups suggested that response precision was significantly lower in the reduced field group (median $=5.01$ ) relative to the control group (median $=13.70, \mathrm{z}=3.67, p<.001$ ) and the reduced central group (median $=11.30, \mathrm{z}=2.88, p=.004$ ). An ANOVA on response times (Figure 6B) showed main effects of both group $\left(\mathrm{F}(2,27)=4.15, p_{p}=\right.$ $\left..034, \eta_{\mathrm{p}}^{2}=.24\right)$ and condition $\left(\mathrm{F}(2,54)=19.03, p_{p}<.001, \eta_{\mathrm{p}}^{2}=.41\right)$, and follow-up tests showed that response times were slower in the reduced field group (median $=7.03 \mathrm{~s}$ ) relative to control (median $=4.86 \mathrm{~s}, \mathrm{z}=4.12, p<.001)$ and reduced central (median $=$ $5.54 \mathrm{~s}, \mathrm{z}=2.65, p=.008$ ). Response times were also significantly slower across all groups in the rectangular room conflict condition (median $=6.59 \mathrm{~s}$ ) compared to the rectangular room + blue wall condition (median $=6.50 \mathrm{~s}, \mathrm{z}=2.32, p=.020$ ) and the square room + blue wall condition (median $=4.75 \mathrm{~s}, \mathrm{z}=3.70, p<.001$ ). However, the difference between the median response times in the two rectangular room conditions was quite small $(0.09 \mathrm{~s})$.
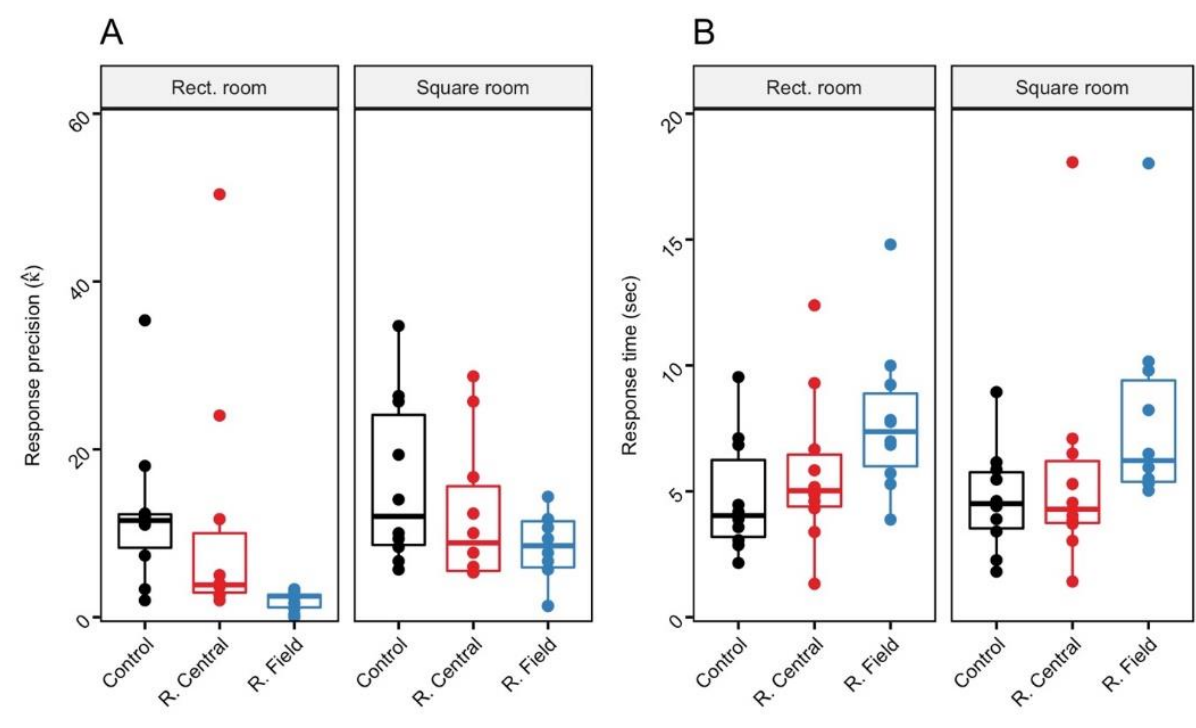

Fig. 5. Results from the rectangular and square room conditions in Experiment 1. A. Response precision is plotted for each experiment group. Circles represent individual participants, box plots denote the $25^{\text {th }}, 50^{\text {th }}$, and $75^{\text {th }}$ quantiles, and whiskers denote the largest and smallest values that are within 1.5 times the inter-quartile range. B. Response time in seconds is plotted in the same

manner as panel A. Rectangular is abbreviated to rect, and reduced is abbreviate to R.

Slower response times in the rectangular room conflict condition likely reflect the added challenge of determining how to respond when the visual environment had changed. As a reminder, in this condition, the location of the painted wall changed between the Learning and Test phases, putting the geometric information in conflict with this salient feature. We hypothesized that the groups might differ in their response strategy, particularly when faced with conflicting cues. We examined this hypothesis by asking whether the weight given to the feature $\left(w_{b}\right)$ differed across groups and conditions (Equation 2). However, the estimated weight that each group gave to the wall feature did 
not systematically differ as predicted. An ANOVA revealed no main effect of group $\left(\mathrm{F}(2,27)=0.75, p_{p}=.48, \eta_{\mathrm{p}}^{2}=.05\right)$. In practice, the variability in weights within each group was very large (Figure 7), particularly in the conflict scenario (rightmost panel). Descriptively, the reduced field and reduced central groups appeared to be more likely to use the salient feature when placed in a cue conflict scenario, but there were large individual differences in strategy that were not well-explained by the current experimental manipulations. There was a significant main effect of condition $(\mathrm{F}(2,54)=$ $16.75, p_{p}<.001, \eta_{\mathrm{p}}^{2}=.38$ ), and follow up tests showed that the feature weight was significantly lower in the rectangular room conflict condition (median $=0.47$ ) as compared to the rectangular room + blue wall (median $=0.84, z=3.24, p=<.001)$ and square room + blue wall (median $=0.84, z=2.67, p=0.004$ ) conditions. This suggests that participants in all groups were more likely to ignore the salient wall when it appeared to be an unreliable, dynamic cue. We also conducted a control experiment exploring the sensitivity of our method to detect differences in response strategy. We substantially reduced the saturation of the blue paint, predicting that this manipulation should reduce the weight on this feature, and recruited 10 additional participants. The results (not shown) suggest that individual differences in response strategy were still quite large with this manipulation. Thus, individual differences in baseline strategy pose a challenge for detecting reliable differences between groups in the current paradigm, but present an interesting avenue for future work.

A

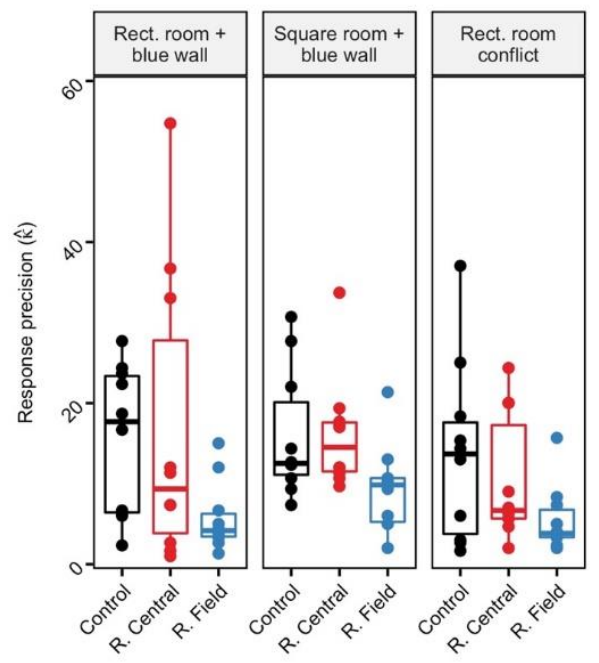

B

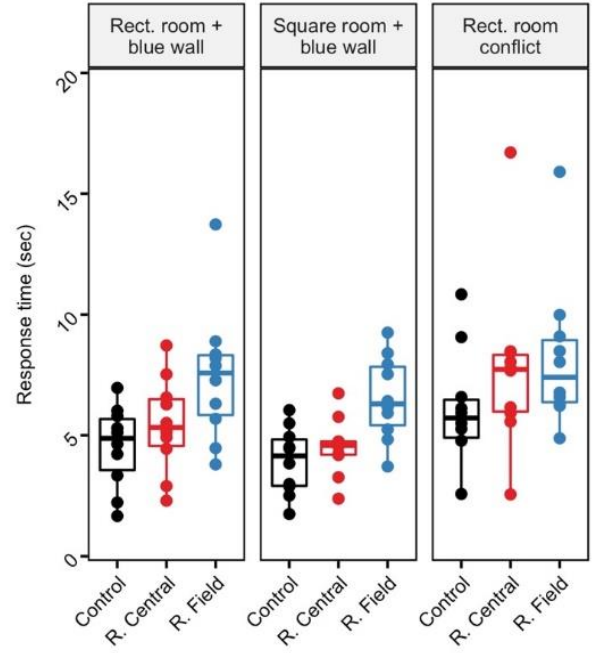

Fig. 6. Results from the rectangular room + blue wall, square room + blue wall, and rectangular room conflict conditions. Data are plotted in the same manner as Figure 5. Rectangular is abbreviated to rect., and reduced is abbreviate to $\mathrm{R}$.

Summary. The results of Experiment 1 suggest that reduced visual field negatively affects precision and response times for reorientation in different environments, more so than a substantial reduction in central visual acuity/contrast sensitivity. When presented with conflicting cues, we observed large individual differences in reliance on environmental information, however the conflict scenario in this experiment was also 
highly artificial (paint moved on a wall). Thus, in Experiment 2 we tested a more naturalistic situation of environmental variability due to changes in lighting.
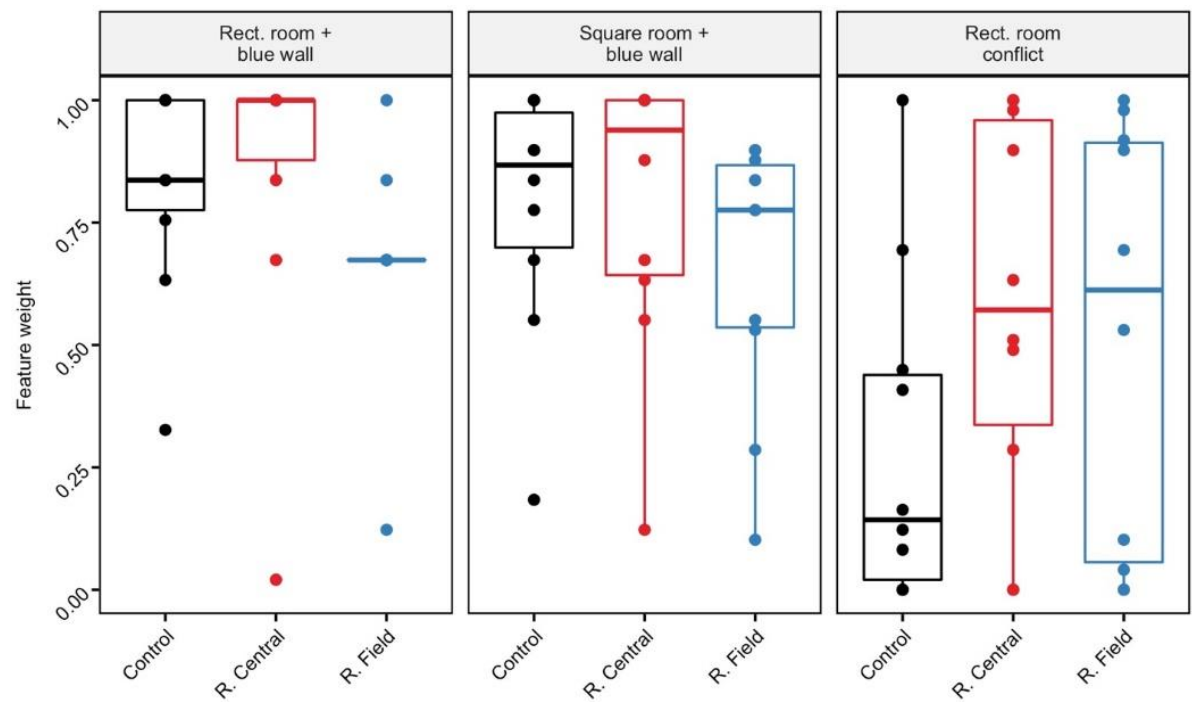

Fig. 7. Response strategy differences between participants. Data are plotted in the same manner as Figure 6, except the ordinate shows the relative weight given to the salient wall feature in the best fit model. Rectangular is abbreviated to rect., and reduced is abbreviate to R.

\section{Experiment 2}

Participants performed the same task as Experiment 1, however, this time the virtual room was rendered in a more realistic design and had windows, furniture, and wall hangings (Figure 2B). Depending on the experimental condition, the room could either be lit by ceiling lights or by sunlight coming through a window.

\subsection{Methods}

Participants. Thirty adults (mean age: 19.78 years, 17 female) completed the study, all with normal or corrected-to-normal visual acuity. Participants were pseudo-randomly assigned into the same groups as in Experiment 1: control (mean age: 19.80 years, 6 female), reduced central (mean age: 19.20 years, 4 female), and reduced field (mean age: 20.05 years, 7 female). One participant declined to indicate their age. The apparatus and procedure were the same as in Experiment 1.

Conditions. Each participant completed 20 trials in each of three different conditions, presented in pseudo-randomized order (60 trials total). In half of the trials, participants were in a rectangular room and in the other half participants were in a square room. Because Experiment 1 revealed no differences between the room shapes, we collapsed across these variables when analyzing the data.

Depending on the experimental condition, participants completed Learning and Test phases in either the same or a different lighting setup (Figure 2B, Table 1). In the ceiling light and sunlight conditions, the room was always lit in the same way. The ceiling light 
came from a set of square lights placed in a symmetrical grid. The sunlight shone through one window, casting a clearly visible highlight on one of the walls. In the dynamic light condition, the lighting changed from ceiling to sun between Learning and Test, or vice-versa.

Table 1. Overview of experimental conditions in Experiment 2

\begin{tabular}{llll}
\hline Condition & Room & Learning lighting & Test lighting \\
\hline Ceiling light & Square/rectangular & Ceiling & Ceiling \\
Sunlight & Square/rectangular & Sun & Sun \\
Dynamic light & Square/rectangular & Ceiling/sun & Sun/ceiling \\
\hline
\end{tabular}

Data analysis. A one-way ANOVA showed no significant differences between the three experimental groups in the $\operatorname{SBSOD}\left(\mathrm{F}(2,27)=1.48, \mathrm{p}=.246, \eta_{\mathrm{p}}^{2}=.10\right)$. The average score was $3.99(\mathrm{sd}=0.92)$. Data were analyzed as described for Experiment 1 . For the ceiling light condition, $\mathrm{A}=\left\{0^{\circ}, 180^{\circ}\right\}$ because the room decorations were symmetric. For the sunlight condition, a second cue was added at $\mathrm{B}=\left\{0^{\circ}\right\}$ to reflect the added highlight on one wall. Since this feature could not be used reliability in the dynamic light condition, $w_{b}$ was fixed at zero for this condition.
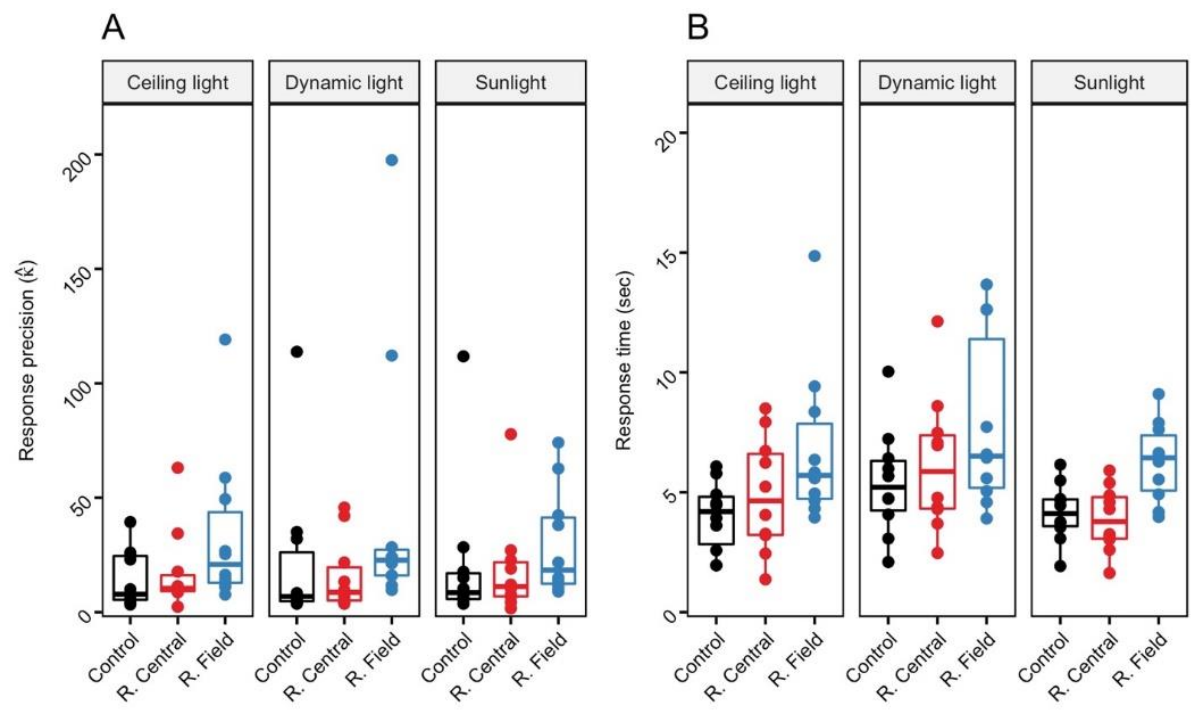

Fig. 8. Results from Experiment 2. All data are plotted in the same manner as Figure 5. Note that the $\mathrm{y}$-axis range in panel A is larger than for Experiment 1. Reduced is abbreviate to R.

Results. In this experiment, we did not observe a significant main effect of group on response precision in the $\operatorname{ANOVA}\left(F(2,27)=1.73, p_{p}=.210, \eta_{\mathrm{p}}^{2}=.11\right)$. In fact, the reduced field group, which had lower precision in Experiment 1, had descriptively 
higher precision on average than the other two groups. We also observed no main effect of condition on response precision $\left(F(2,54)=0.56, p_{p}=.882, \eta_{\mathrm{p}}^{2}=.02\right)$. However, the response time results were more similar to Experiment 1. An ANOVA on response times showed a significant main effect of both group $\left(F(2,27)=3.82, p_{p}=.038, \eta_{\mathrm{p}}^{2}=.22\right)$ and condition $\left(F(2,54)=13.17, p_{p}<.001, \eta_{\mathrm{p}}^{2}=.33\right)$. Follow up tests showed that the response times were slower in the reduced field group (median $=6.32 \mathrm{~s})$, relative to both the control (median $=4.51 \mathrm{~s}, z=3.64, p<.001$ ) and reduced central groups $($ median $=$ $4.48 \mathrm{~s}, z=2.96, p=.003$ ). In addition, response times across groups were slower in the dynamic light condition (median $=5.83 \mathrm{~s}$ ) as compared to the sunlight condition (median $=4.78 \mathrm{~s}, z=2.25, p=.024$ ). These results highlight the importance of environmental factors and cues during reorientation. The response times suggest that reduced visual field increased the challenge associated with reorienting oneself. However, perhaps the more numerous visual features available in these rooms allowed precision to be high despite the increased challenge. All groups, including controls, took more time to perform the task if the lighting changed, even though the physical features and geometry of the room were constant. This finding suggests that even highly common scene dynamics such as lighting can influence reorientation performance.

\section{Discussion}

Here, we reported on two VR experiments exploring how simulated vision reduction influences reorientation performance. The most consistent result observed across experiments was an increase in the time taken to reorient in participants with simulated reduction in peripheral vision (the reduced field groups). In some conditions, we also observed reduced precision in this group - meaning that participants were less able to reliably recover their desired orientation.

There are a range perceptual changes with reduced vision that may have impacted reorientation performance in our study, including impaired distance or shape estimates (Fortenbaugh et al., 2008, 2007) and impaired detection of environmental features (Bochsler et al., 2013, 2012). Because reorienting requires searching the environment for information, the slower response times may also point to challenges with performing visual search, which are increased when peripheral vision is limited (Senger et al., 2017). Richer types of environments (such as those used in Experiment 2) may make it easier to ultimately identify the target of search, even if it still takes longer. Indeed, while several visual enhancement devices for navigation have been proposed that increase geometric information or expand the visual field (Hicks et al., 2013; Kinateder et al., 2018; VargasMartín and Peli, 2002), it may be fruitful to consider adapting tools that support visual search, for example, by cuing the location of reliable environmental landmarks (Zhao et al., 2016). By exploring dynamic lighting conditions, we hoped to understand if changes in brightness and contrast could preferentially affect reorientation with reduced vision, however we did not find evidence for consistent effects here.

There are several limitations to the current study. Participants wore goggles that simulated visual impairment and, consequently, never experienced reduced central vision or reduced visual field outside of the experiment. Low vision simulators are often employed to examine task performance in controlled settings (Barhorst-Cates et al., 2017; Bochsler et al., 2012; Dunn and Rushton, 2018; Kallie et al., 2012; Legge et al., 2016a; Rand et al., 2015), but cannot simulate the experience of living with impaired 
vision. Using simulators in this study allowed us to perform between-subject comparisons with control and test groups that were highly similar to each other except for their vision during the task. Moving forward, examining how differing levels and types of genuine visual impairment, training, experience, and other demographics impact spatial reorientation will be important. For example, while the current results did not reveal any differences in strategy in Experiment 1, prior experience and training likely heavily influence individual strategies used. Despite offering high experimental control, current tools for simulation also leave room for improvement. For example, monocular field restriction (with one eye occluded) also removes all stereovision. Similarly, Bangerter occlusion foils both reduce acuity and contrast sensitivity. For research that aims to use simulated vision reduction, advances in eye-tracking and real-time rendering may create new possibilities for precisely simulating specific deficits. We also note that each individual group size in both Experiments 1 and 2 was relatively small for detecting between-group differences $(n=10)$. While our new parametric model provided a good account of each individual participant's dataset, these small sample sizes limit that ability of the current studies to reliably detect the effects of the independent variables on response precision. For example, reduced central vision likely affects reorientation, but the effect for the level simulated here may have been too small to detect with this sample. In future work, we plan to use the new model we developed to examine reorientation performance in larger sample of individuals with real visual impairments.

Moving forward, immersive VR tasks such as reorientation present a promising direction for creating standardized assessments for orientation and mobility with impaired vision. VR provides a good compromise between simulating the complex cues present during natural tasks, but also being deployable in controlled environments at relatively low cost. Interestingly, recent work suggests that mobility and navigation training for visually impaired users in VR transfers to real world (Bowman and Liu, 2017). Moving forward, VR may thus provide an integrated platform for assessing the task performance and training the usage of new assistive tools.

\section{Acknowledgments}

The authors would like to thank Kendrick Kay for helpful discussions on the model fitting procedure, as well as Klara Barbarossa and Alaa Mustafa for their help with data collection.

\section{References}

Barhorst-Cates, E.M., Rand, K.M., Creem-Regehr, S.H. (2017). Let me be your guide: physical guidance improves spatial learning for older adults with simulated low vision. Experimental Brain Research 235, 3307-3317. https://doi.org/10.1007/s00221-017-5063-8

Barhorst-Cates, E.M., Rand, K.M., Creem-Regehr, S.H. (2016). The effects of restricted peripheral field-of-view on spatial learning while navigating. PLOS ONE 11, 1-19. https://doi.org/10.1371/journal.pone.0163785

Berens, P. (2009). CircStat: A MATLAB toolbox for circular statistics. Journal of Statistical Software 31 .

Black, A., Lovie-Kitchin, J.E., Woods, R.L., Arnold, N., Byrnes, J., Murrish, J. (1997). Mobility performance with retinitis pigmentosa. Clinical and Experimental Optometry 80, 1-12. https://doi.org/10.1111/j.1444-0938.1997.tb04841.x 
Bochsler, T.M., Legge, G.E., Gage, R., Kallie, C.S. (2013). Recognition of ramps and steps by people with low vision. Investigative Ophthalmology and Visual Science 54, 288-294. https://doi.org/10.1167/iovs.12-10461

Bochsler, T.M., Legge, G.E., Kallie, C.S., Gage, R. (2012). Seeing steps and ramps with simulated low acuity. Optometry and Vision Science 89, E1299-E1307. https://doi.org/10.1097/opx.0b013e318264f2bd

Bourne, R.R.A., Flaxman, S.R., Braithwaite, T., Cicinelli, M. V, Das, A., Jonas, J.B., Keeffe, J., Kempen, J.H., Leasher, J., Limburg, H., Naidoo, K., Pesudovs, K., Resnikoff, S., Silvester, A., Stevens, G.A., Tahhan, N., Wong, T.Y., Taylor, H.R., Vision Loss Expert Group (2017) Magnitude, temporal trends, and projections of the global prevalence of blindness and distance and near vision impairment: a systematic review and meta-analysis. The Lancet. Global health 5, e888-e897. https://doi.org/10.1016/S2214-109X(17)30293-0

Bowman, E.L., Liu, L. (2017). Individuals with severely impaired vision can learn useful orientation and mobility skills in virtual streets and can use them to improve real street safety. PLoS ONE 12, e0176534. https://doi.org/10.1371/journal.pone.0176534

Brown, B., Brabyn, L., Welch, L., Haegerstrom-Portnoy, G., Colenbrander, A. (1986). Contribution of vision variables to mobility in age-related maculopathy patients. Optometry and Vision Science. https://doi.org/10.1097/00006324-198609000-00006

Burgess, N. (2006). Spatial memory: how egocentric and allocentric combine. Trends in Cognitive Sciences 10, 551-557. https://doi.org/10.1016/j.tics.2006.10.005

Chan, T., Friedman, D.S., Bradley, C., Massof, R. (2018). Estimates of incidence and prevalence of visual impairment, low vision, and blindness in the United States. JAMA Ophthalmology 136, 12-19. https://doi.org/10.1001/jamaophthalmol.2017.4655

Cheng, K. (1986). A purely geometric module in the rat's spatial representation. Cognition 23, 149-178. https://doi.org/10.1016/0010-0277(86)90041-7

Cheng, K., Huttenlocher, J., Newcombe, N.S. (2013). 25 years of research on the use of geometry in spatial reorientation: A current theoretical perspective. Psychonomic Bulletin and Review 20, 1033-1054. https://doi.org/10.3758/s13423-013-0416-1

Cheung, A., Stürzl, W., Zeil, J., Cheng, K. (2008). The information content of panoramic images II: View-based navigation in nonrectangular experimental arenas. Journal of Experimental Psychology: Animal Behavior Processes 34, 15-30. https://doi.org/10.1037/00977403.34.1.15

Daga, F.B., Macagno, E., Stevenson, C., Elhosseiny, A., Diniz-Filho, A., Boer, E.R., Schulze, J., Medeiros, F.A. (2017). Wayfinding and glaucoma: A virtual reality experiment. Investigative Ophthalmology and Visual Science 58, 3343-3349. https://doi.org/10.1167/iovs.17-21849

Dinno, A. (2017). Package "dunn.test." $R$ package version 1.3.5.

Dunn, M.J., Rushton, S.K. (2018). Lateral visual occlusion does not change walking trajectories. Journal of Vision 18, 11. https://doi.org/10.1167/18.9.11

Epstein, R.A., Patai, E.Z., Julian, J.B., Spiers, H.J. (2017). The cognitive map in humans: Spatial navigation and beyond. Nature Neuroscience. https://doi.org/10.1038/nn.4656

Fortenbaugh, F.C., Hicks, J.C., Hao, L., Turano, K.A. (2007). Losing sight of the bigger picture: Peripheral field loss compresses representations of space. Vision Research 47, 2506-2520. https://doi.org/10.1016/j.visres.2007.06.012

Fortenbaugh, F.C., Hicks, J.C., Turano, K.A. (2008). The effect of peripheral visual field loss on representations of space: Evidence for distortion and adaptation. Investigative Ophthalmology and Visual Science 49, 2765-2772. https://doi.org/10.1167/iovs.07-1021

Hassan, S.E., Lovie-Kitchin, J.E., Woods, R.L. (2002). Vision and mobility performance of subjects with age-related macular degeneration. Optometry and Vision Science 79, 697-707. https://doi.org/10.1097/00006324-200211000-00007

Hassan, S.E., Lovie-Kitchin, J.E., Woods, R.L., Soong, G.P.Y. (1999). Orientation and mobility in age-related macular degeneration. Visual Impairment Research 1, 175-180. https://doi.org/10.1076/vimr.1.3.175.4442 
Hegarty, M., Richardson, A.E., Montello, D.R., Lovelace, K., Subbiah, I. (2002). Development of a self-report measure of environmental spatial ability. Intelligence 30, 425-447. https://doi.org/10.1016/S0160-2896(02)00116-2

Hermer, L., Spelke, E. (1996). Modularity and development: The case of spatial reorientation. Cognition 61, 195-232. https://doi.org/10.1016/S0010-0277(96)00714-7

Hermer, L., Spelke, E.S. (1994). A geometric process for spatial reorientation in young children. Nature 370, 57-59. https://doi.org/10.1038/370057a0

Hicks, S.L., Wilson, I., Muhammed, L., Worsfold, J., Downes, S.M., Kennard, C. (2013). A depthbased head-mounted visual display to aid navigation in partially sighted individuals. PLoS ONE 8, 1-8. https://doi.org/10.1371/journal.pone.0067695

Huang, J., Kinateder, M., Dunn, M.J., Jarosz, W., Yang, X.D., Cooper, E.A. (2019). An augmented reality sign-reading assistant for users with reduced vision. PLOS ONE 14, 1-9. https://doi.org/10.1371/journal.pone.0210630

Kalia, A.A., Legge, G.E., Giudice, N.A. (2008). Learning building layouts with non-geometric visual information: The effects of visual impairment and age. Perception 37, 1677-1699. https://doi.org/10.1068/p5915

Kallie, C.S., Legge, G.E., Yu, D. (2012). Identification and detection of simple 3D objects with severely blurred vision. Investigative Ophthalmology and Visual Science 53, 7997-8005. https://doi.org/10.1167/iovs.12-10013

Kinateder, M., Gualtieri, J., Dunn, M.J., Jarosz, W., Yang, X.D., Cooper, E.A. (2018). Using an augmented reality device as a distance-based vision aid-Promise and limitations. Optometry and Vision Science 95, 727-737. https://doi.org/10.1097/OPX.0000000000001232

Kuyk, T., Elliott, J.L. (1999). Visual factors and mobility in persons with age-related macular degeneration. Journal of Rehabilitation Research and Development 36, 303-312.

Legge, G.E., Gage, R., Baek, Y., Bochsler, T.M. (2016a). Indoor spatial updating with reduced visual information. PLOS ONE 11, 1-22. https://doi.org/10.1371/journal.pone.0150708

Legge, G.E., Granquist, C., Baek, Y., Gage, R. (2016b). Indoor spatial updating with impaired vision. Investigative Ophthalmology and Visual Science 57, 6757-6765. https://doi.org/10.1167/iovs.16-20226

Lovie-Kitchin, J.E., Soong, G.P., Hassan, S.E., Woods, R.L. (2010). Visual field size criteria for mobility rehabilitation referral. Optometry \& Vision Science 87, 948-957.

Marron, J.A., Bailey, I.L. (1982). Visual factors and orientation-mobility performance. Optometry and Vision Science 59, 413-426. https://doi.org/10.1097/00006324-198205000-00009

Rand, K.M., Creem-Regehr, S.H., Thompson, W.B. (2015). Spatial learning while navigating with severely degraded viewing: The role of attention and mobility monitoring. Journal of Experimental Psychology: Human Perception and Performance 41, 649-664. https://doi.org/10.1037/xhp0000040

Ratliff, K.R., Newcombe, N.S. (2008). Is language necessary for human spatial reorientation? Reconsidering evidence from dual task paradigms. Cognitive Psychology 56, 142-163. https://doi.org/10.1016/j.cogpsych.2007.06.002

Senger, C., Rita, M., Alves, R., Gustavo, C., Moraes, D., Issa, L., Fendi, D. (2017). Visual Search Performance in Patients with Vision Impairment: A Systematic Review. Current Eye Research 42, 1561-1571. https://doi.org/10.1080/02713683.2017.1338348

Stürzl, W., Cheung, A., Cheng, K., Zeil, J. (2008). The information content of panoramic images I: The rotational errors and the similarity of views in rectangular experimental arenas. Journal of Experimental Psychology: Animal Behavior Processes 34, 1-14. https://doi.org/10.1037/0097-7403.34.1.1

Turano, K.A., Broman, A.T., Bandeen-Roche, K., Munoz, B., Rubin, G.S., West, S.K. (2004). Association of visual field loss and mobility performance in older adults: Salisbury eye evaluation study. Optometry and Vision Science 81, 298-307. https://doi.org/10.1097/01.opx.0000134903.13651.8e

Twyman, A.D., Holden, M.P., Newcombe, N.S. (2018). First direct evidence of cue integration in reorientation: A new paradigm. Cognitive Science 42, 923-936. https://doi.org/10.1111/cogs.12575 
Vargas-Martín, F., Peli, E. (2002). Augmented-view for restricted visual field: Multiple device implementations. Optometry and Vision Science 79, 715-723.

https://doi.org/10.1097/00006324-200211000-00009

Weiner, W.R., Welsh, R.L., Blasck, B.B. (2018). Foundations of Orientation and Mobility Volume 1: History and Theory, 3rd ed. AFB Press.

Wheeler, B., Torchuano, M. (2016). Package "lmPerm." $R$ package version 2.1.0.

Xu, Y., Regier, T., Newcombe, N.S. (2017). An adaptive cue combination model of human spatial reorientation. Cognition 163, 56-66. https://doi.org/10.1016/j.cognition.2017.02.016

Zhao, Y., Szpiro, S., Knighten, J., Azenkot, S. (2016). CueSee: Exploring visual cues for people with low vision to facilitate a visual search task, in: UbiComp 2016 - Proceedings of the 2016 ACM International Joint Conference on Pervasive and Ubiquitous Computing. pp. $73-$ 84. https://doi.org/10.1145/2971648.2971730

Received July 29, 2021, accepted August 7, 2021 